This is a self-archived version of an original article. This version may differ from the original in pagination and typographic details.

Author(s): Sheik, Muhammad Usman; Asghar, Muhammad Zeeshan; Jäntti, Riku

Title: Dual Connectivity in Non-Stand Alone Deployment mode of $5 \mathrm{G}$ in Manhattan Environment

Year: 2020

Version: Accepted version (Final draft)

Copyright: @ 2020 IEEE

Rights: In Copyright

Rights url: http://rightsstatements.org/page/InC/1.0/?language=en

Please cite the original version:

Sheik, M. U., Asghar, M. Z., \& Jäntti, R. (2020). Dual Connectivity in Non-Stand Alone Deployment mode of 5 G in Manhattan Environment. In ICEIC 2020 : Proceedings of the 19th International Conference on Electronics, Information and Communications, Barcelona, Spain (pp. 1-4). IEEE. https://doi.org/10.1109/ICEIC49074.2020.9051202 


\title{
Dual Connectivity in Non-Stand Alone Deployment mode of 5G in Manhattan Environment
}

\author{
Muhammad Usman Sheikh*, Muhammad Zeeshan Asghar ${ }^{\dagger}$ and Riku Jäntti* \\ *Department of Communications and Networking, Aalto University, Espoo, 02150 Finland. \\ Email: \{muhammad.sheikh; riku.jantti\}@aalto.fi \\ $\dagger^{\dagger}$ Faculty of Information Technology, University of Jyväskylä, Finland. \\ Email: muhammad.z.asghar@jyu.fi
}

\begin{abstract}
The main target of this paper is to analyze the performance of an outdoor user in a dense micro cellular Manhattan grid environment using a ray launching simulation tool. The radio propagation simulations are performed using a Shoot and Bouncing Ray (SBR) method. The network performance is analyzed at three different frequencies i.e. $1.8 \mathrm{GHz}$, 3.5 GHz, and $28 \mathrm{GHz}$. Additionally, the benefits of combining LTE and potential $5 \mathrm{G}$ frequency bands by using feature of Dual Connectivity (DC) in an outdoor scenario has been highlighted. The considered performance metrics are received signal level, SINR, application throughput. The acquired simulation results from Manhattan canyon street environment reveal that a good 5G outdoor coverage can be provided at $3.5 \mathrm{GHz}$ and $28 \mathrm{GHz}$ while using existing 4G micro sites. The impact of Dual Connectivity at user's throughput is studied in this article, and it is shown user throughput can be doubled by leveraging the benefits of LTE and 5G NR together.
\end{abstract}

Index Terms-Dual connectivity; LTE; Ray tracing; 5G; System performance.

\section{INTRODUCTION}

The demand for high data rate and large data volume consumption continues to rise. One intuitive solution to fulfill the demand of high capacity and high data rate is to acquire more spectrum which is available at mid and high frequency bands i.e., at $3.5 \mathrm{GHz}$ and $28 \mathrm{GHz}$, respectively. These bands have high potential for $5 \mathrm{G}$, but there are certain challenges associated with them. The Millimeter Wave (mmWave) bands offer order of magnitude more free spectrum as compared with the current cellular frequency bands i.e., below $3 \mathrm{GHz}$. However, providing ubiquitous coverage and robust un-interrupted services in Non Line of Sight (NLOS) condition is challenging in mmWave case due to heavy blockage caused by obstacles and common building materials [1], [2]. The other possible solution for capacity crunch is to increase the density of the micro or small cells, and increase the reuse of existing spectrum. In dense network deployment, the capacity of the network is enhanced by higher frequency reuse in small area. However, the increase in the system capacity is not linear with the increase in the density of the cells [3].

Carrier Aggregation (CA) is a way to increase the bandwidth available for individual users. The concept of $\mathrm{CA}$ was first introduced in the Release 10 of $3^{\text {rd }}$ Generation Partner Project (3GPP) specification as a part of LTE Advanced (LTE-A) for enhancing the user throughput by utilizing/aggregating upto five contiguous or non-contiguous carriers. Later in Release

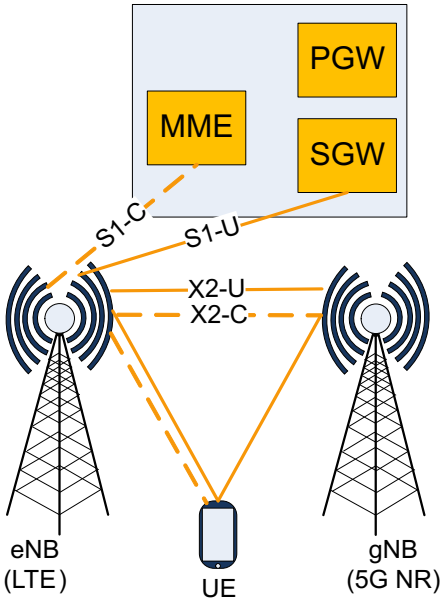

Fig. 1. Illustration of Non-Stand Alone (NSA) deployment of 5G with 3C architecture configuration.

12 of 3GPP, the aggregation between TDD and FDD modes of LTE system was allowed, and the Release 13 of 3GPP allowed the aggregation of upto 32 carriers. Whereas, the idea of Dual Connectivity (DC) introduced in Release 15 3GPP is envisioned as a promising technique for $5 \mathrm{G}$ system to enhance the user throughput. The concept of dual connectivity in 5G is similar to CA in LTE, however, it is different from conventional carrier aggregation as it allows the user to utilize the resources from two different Radio Access Technologies (RATs) i.e. LTE and 5G New Radio (NR) which are operating at two different frequencies. In dual connected mode the UE leverages benefits from both the LTE and 5G system, and the UE is connected to the LTE eNodeB (LTE-eNB) a.k.a Master eNB (MeNB) and 5G gNodeB (5G-gNB) a.k.a Secondary eNB (SeNB) simultaneously as shown in Fig .1. The LTE and 5G NR layers are aggregated via X2 interface between LTE eNB and 5G NR gNB [4]. One of the objective of the dual connectivity configuration is to provide system reliability for control information by utilizing the LTE coverage from the lower band and diminish the coverage interruptions that might occur at mid band or high band i.e. $28 \mathrm{GHz}$.

Applications with high throughput requirement can make use of both the layers at the same time in order to increase the system bandwidth and thus the throughput of the user. By 
using dual connectivity feature it is possible to use both the LTE and the 5G NR for user plane traffic, or LTE can be used for control plane and 5G NR for the user plane traffic. Another reason behind the LTE and 5G tight integration is the economic aspect. Dual connectivity can be used as an impressive tool to improve the mobility and robustness of the 5G system operating at mmWave bands [5]. Dual connectivity requires strong inter-working between LTE and 5G NR which is easy to achieve in case of co-located LTE and 5G NR base station. Initially, it is expected to have $5 \mathrm{G}$ roll-out in a NonStand Alone (NSA) mode which means that the existing LTE infrastructure will be used for establishing a 5G connection. The LTE-5G tight integration architecture for dual connectivity in NSA mode is shown in Fig. 1, it is also frequently called as "architecture configuration 3" [4], [6]. In this configuration, there is a bearer split at MeNB, and therefore there exists only a single bearer for each UE in dual connectivity mode, and the flow split occurs at MeNB. In case of Stand Alone (SA) 5G deployment, the DC can exploit the transmission diversity by sending the same information from multiple RATs to enhance the system reliability or by sending independent bit streams from two RATs to increase the user throughput [4]. However, we have only targeted the NSA deployment of 5G NR in this paper.

By means of sophisticated and comprehensive 3D ray tracing simulations, the target of this study is to evaluate the feasibility of deploying 5G in mid and high band at existing $4 \mathrm{G}$ sites in dense Manhattan environment. The other target is to analyze the user performance utilizing the feature of dual connectivity. The remainder of this paper is as follows. Section II deals with the simulation methodology and discusses about simulation environment, tool and the simulation cases. Section III presents and evaluates the results, and finally the Section IV concludes the paper.

\section{Simulation Methodology}

This section describes about the simulation methodology and provides detail about the simulation tool, simulation environment, and explains the considered cases.

\section{A. Simulation environment description}

A dense urban environment is targeted in this study. Therefore, we have considered a Manhattan city grid model for simulations as proposed in [7]. The Fig. 2 illustrates a simulation environment where each square represents a block of buildings with $100 \mathrm{~m} \times 100 \mathrm{~m}$ dimension, and all the buildings have $30 \mathrm{~m}$ height. Two consecutive building blocks are separated by $30 \mathrm{~m}$ wide road. On the road, there is a two way traffic and a pedestrian pathway of $3 \mathrm{~m}$ wide on both sides of the road. Unlike macro cell deployment above the average roof tops, the micro cell is generally deployed on the building wall and is placed under the average roof top. Micro cells have comparatively low transmission power and have relatively small coverage area compared with the macro cell. The layout of micro sites follows a regular grid. The Manhattan area under consideration is covered with nineteen micro sites, where each

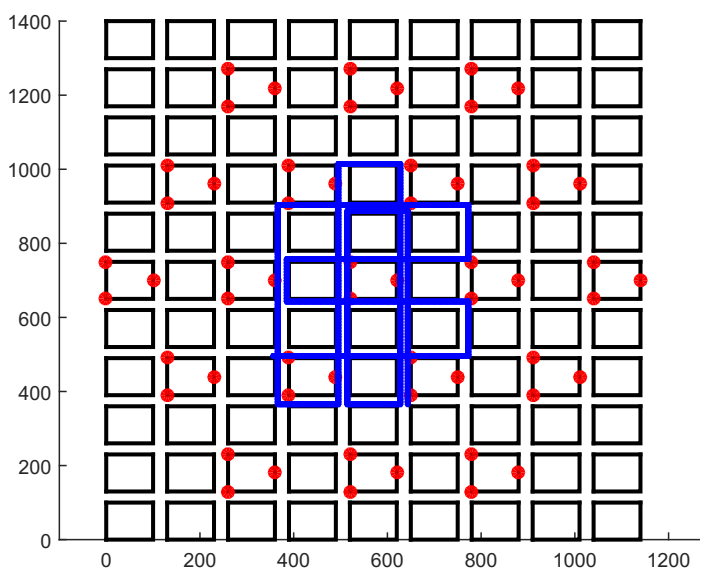

Fig. 2. Simulation environment along with location of micro base stations.

micro site has three micro cells as shown in Fig. 2. The three cells of micro sites have an azimuth of 0 degree, 135 degree, and 225 degree. The antennas of all micro cells are placed at a height of $25 \mathrm{~m}$ which is clearly below the average building height of $30 \mathrm{~m}$. The maximum transmission power for micro cell is limited to $33 \mathrm{dBm}$ (2 watts).

\section{B. Simulation Tool and Simulation Models}

The MATLAB is used as a simulation platform for coverage prediction and as well as for analyzing the performance of handover. A Shoot and Bouncing Ray (SBR) method based ray launching tool is developed by the authors in MATLAB for finding the propagation paths between the transmitter and the receiver with the given number of reflections and diffraction. A ray launching tool considers a Line of Sight (LOS) path, penetrated path through the walls, and paths with multiple reflections and diffraction. In a ray launching tool, the rays are launched with $0.5^{\circ}$ angular separation between two consecutive rays from the transmitter. Where, each ray continues to propagate until it reaches the maximum allowed number of interactions/reflections. The maximum number of supported reflection is ten in our simulations. In a Manhattan type environment, it is critical to consider diffracted paths coming from the edges/corners of the building. A penetrated path through the buildings is also included. However, an open space without any partition wall is assumed for the indoor plan. Therefore, a penetrated path takes into account only the Building Penetration Loss (BPL). In this study, modern buildings are considered which are assumed to have $70 \%$ of the IRR glass windows and $30 \%$ of the concrete wall. The frequency and material dependent BPL model provided at [8] is used for estimating penetration loss for penetrated paths. The effect of shadowing is generally modeled by lognormal distribution. As we have considered user mobility, there exists a time correlation and the fading values at the adjacent distances are correlated. The parameters and models related to shadowing and auto-correlation of shadowing given in [9] are used in our simulations. Car traffic is not modeled in this study. Here only an outdoor user moving with $3 \mathrm{~km} / \mathrm{h}$ 
and $40 \mathrm{~km} / \mathrm{h}$ is considered; therefore an indoor floor plan is neglected. In our simulations, the handover events are triggered based on combine Reference Signal Received Power (RSRP) and Signal to Interference plus Noise Ratio (SINR) values. An offset of $3 \mathrm{~dB}$ is used for both RSRP and SINR to avoid the ping pong effect during the handover. The extended 3GPP antenna model presented in [9] is used to model the antenna radiation pattern in azimuth and elevation domain. The antenna radiation modeling parameters i.e. Half Power Beamwidth (HPBW) in horizontal domain $\left(\theta_{H}\right)$, HPBW in vertical domain $\left(\theta_{V}\right)$, Front to Back ratio in horizontal domain $\left(F B R_{H}\right)$, Side Lobe Level in vertical domain $\left(S L L_{V}\right)$, and antenna maximum gain $\left(A_{M}\right)$ are provided in the Table. I.

TABLE I

EXTENDED 3GPP ANTENNA MODEL PARAMETERS

\begin{tabular}{|c|c|c|c|c|}
\hline$\theta_{H}$ & $\begin{array}{c}\theta_{V} \\
{\left[^{\circ}\right]}\end{array}$ & $\begin{array}{c}F B R_{H} \\
{\left[{ }^{\circ}\right]}\end{array}$ & $\begin{array}{c}S L L_{V} \\
{[d B]}\end{array}$ & $\begin{array}{c}A_{M} \\
{[d B i]}\end{array}$ \\
\hline 65 & 7 & 30 & -18 & 17.7 \\
\hline
\end{tabular}

\section{Simulation cases and performance metrics}

This campaign of simulation targets a dense outdoor micro cell deployment only. The frequency of $1.8 \mathrm{GHz}$ is currently used for LTE system, whereas the $3.5 \mathrm{GHz}$ and $28 \mathrm{GHz}$ bands are the potential bands for $5 \mathrm{G}$ system. Therefore, the system performance is individually analyzed at $1.8 \mathrm{GHz}, 3.5 \mathrm{GHz}$, and $28 \mathrm{GHz}$, and the impact of dual connectivity on user throughput is analyzed separately. In terms of interference, a worst case scenario is considered here i.e., all the $4 \mathrm{G}$ and $5 \mathrm{G}$ base stations are transmitting at their full power without any power control. The performance of network is analyzed in terms of received signal power, SINR, and user application throughput. Mobility effect is taken into account while analyzing the different performance metrics. Handover rate is used to quantify the handover delay per unit time or Handover Cost $\left(H O_{\text {Cost }}\right)$. The handover delay per unit time is a function of Handover Rate $\left(H O_{\text {Rate }}\right)$ and Handover Delay $\left(H O_{\text {Delay }}\right)$ as expressed in Eq. 1 . A handover delay of $2 \mathrm{~s}$ is used in this paper.

$$
H O_{\text {Cost }}=H O_{\text {Rate }} * H O_{\text {Delay }}
$$

Throughput is the direct function of the user's SINR and the Bandwidth $(B)$ utilization. For a user throughput we take into account the overhead caused by the $\mathrm{HO}$ and also consider the Control Overhead $(\alpha)$ caused by the control signalling as shown in Eq. 2. A control overhead of 0.3 is assumed.

$$
T=B * \log _{2}(1+S I N R)\left(1-H O_{\text {Cost }}\right)(1-\alpha)
$$

\section{Simulation Results AND Discussion}

This section provides the simulation results and discusses about the performance comparison between different considered cases. The first metric discussed here is the received power. The Fig. 3 shows the CDF plots of downlink received power. In a considered dense microcellular network the outdoor user stays mostly in a LOS with the serving base station.

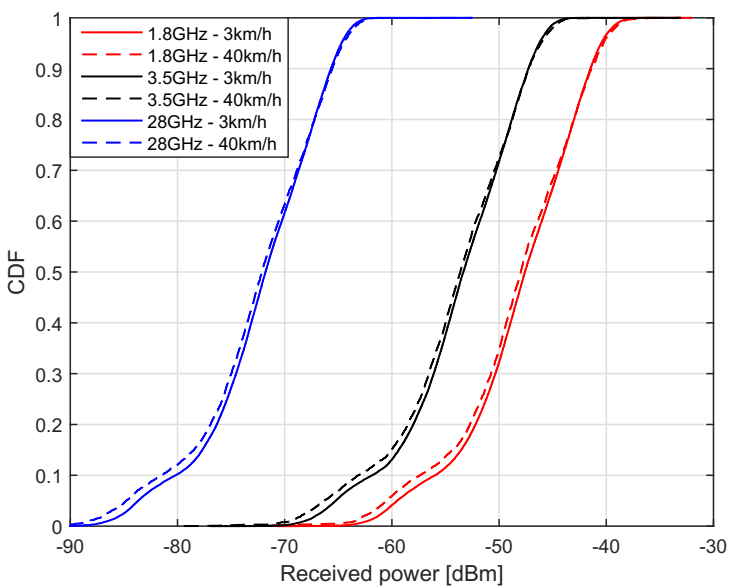

Fig. 3. CDF of received power.

The acquired results show that a mean received power of $48.5 \mathrm{dBm},-54.2 \mathrm{dBm}$, and $-72.6 \mathrm{dBm}$ is attained at $1.8 \mathrm{GHz}$, $3.5 \mathrm{GHz}$, and $28 \mathrm{GHz}$, respectively. It means that there is a difference of $5.7 \mathrm{~dB}$ and $24.1 \mathrm{~dB}$ between the mean received power of $3.5 \mathrm{GHz}$ and $28 \mathrm{GHz}$ with respect to $1.8 \mathrm{GHz}$ band of operation. It is interesting to find that in case of dense microcellular network deployment a healthy received signal strength i.e., coverage can be obtained for outdoor users even at $28 \mathrm{GHz}$ frequency band of operation, and the system is not coverage/noise limited. It shows the suitability of $5 \mathrm{G}$ radio at mid and high bands at existing outdoor dense micro sites for serving outdoor users. The received power results presented in Fig. 3 show that there is a slight drift between the received power of a user moving with $3 \mathrm{~km} / \mathrm{h}$ and $40 \mathrm{~km} / \mathrm{h}$ speed.

The second performance metric considered here is the quality metric i.e. the SINR. The Fig. 4 shows the CDF plots of SINR for different considered cases. There is a large bandwidth available at $28 \mathrm{GHz}$ band, therefore, for the case of $28 \mathrm{GHz}$ the SINR results are provided with $20 \mathrm{MHz}$ and

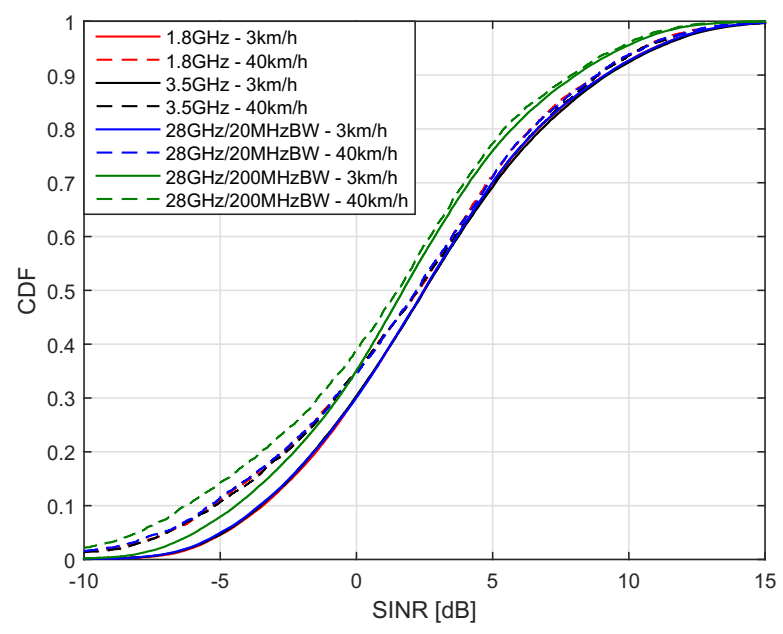

Fig. 4. CDF of signal to interference plus noise ratio. 
$200 \mathrm{MHz}$ system bandwidth. It can be seen in Fig. 4 the SINR CDF curves are overlapping over each other, and the CDF plots are almost identical at $1.8 \mathrm{GHz}, 3.5 \mathrm{GHz}$, and $28 \mathrm{GHz}$ with $20 \mathrm{MHz}$ bandwidth. It shows that at substantially good received power level, the SINR becomes independent of frequency of operation. It means that a $5 \mathrm{G}$ user operating at $3.5 \mathrm{GHz}$ or $28 \mathrm{GHz}$ can enjoy a same Quality of Service (QoS) as 4G LTE user at $1.8 \mathrm{GHz}$, given a good coverage level is maintained. It is found that for slow moving users utilizing $20 \mathrm{MHz}$ bandwidth the mean SINR of about $2.65 \mathrm{~dB}$ is attained at all considered frequencies. The fast moving users had lower SINR i.e. around $1.9 \mathrm{~dB}$ as compared with slow moving mobiles. The large utilization of $200 \mathrm{MHz}$ bandwidth at $28 \mathrm{GHz}$ frequency band further slightly degraded the mean SINR to $1.75 \mathrm{~dB}$ level. It shows that the large bandwidth can be used extensively as a healthy SINR is maintained through the route due to mainly LOS propagation in dense microcellular environment. It also shows that in case of good received power levels even the large noise due to large bandwidth does not affect the SINR significantly. Therefore, it does not restrain the continuous usage of large i.e., $200 \mathrm{MHz}$ bandwidth for the user.

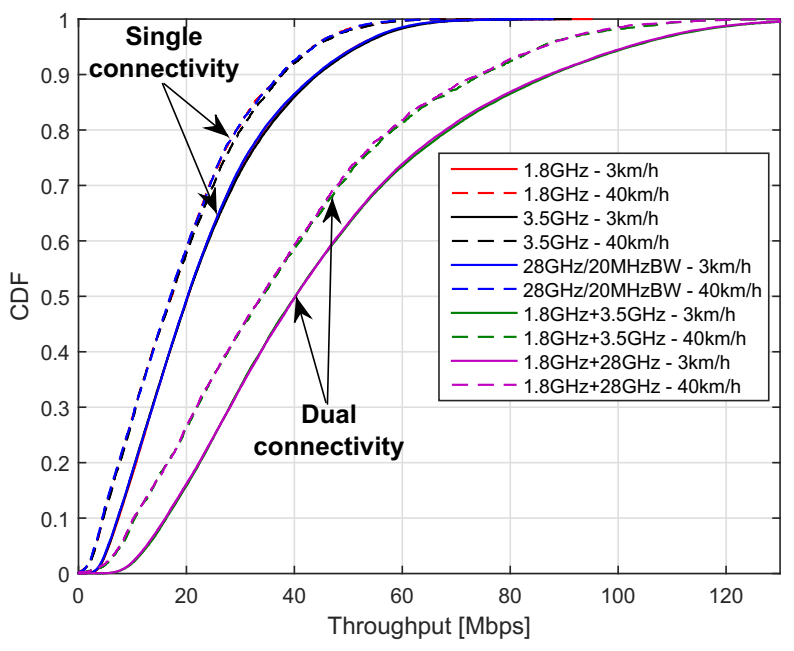

Fig. 5. CDF of application throughput.

Fig. 5 shows the CDF plots of user application throughput. The achieved SINR can be directly translated into spectral efficiency or in other words the throughput of the user. In case of single connectivity to any of the frequency layer with $20 \mathrm{Mhz}$ bandwidth, the achieved mean user throughput for slow moving and fast moving user is around $23.2 \mathrm{Mbps}$ and 19.1 Mbps, respectively. However, in case of dual connectivity the user throughput is doubled to $46.3 \mathrm{Mbps}$ and $38.2 \mathrm{Mbps}$ for slow moving and fast moving users, respectively. From the throughput results presented in Fig. 5 it can be observed that in NSA deployment of dense $5 \mathrm{G}$ microcellular network, the feature of dual connectivity improves the user experience and throughput by a fairly significant margin. It highlights the fact that adding a 5G NR at existing LTE site is an effective and cost efficient way of improving the user experience, and it eludes the need of site densification. The large bandwidth available at high band i.e., $28 \mathrm{GHz}$ can provide further higher data rates and additional capacity on top of the combined dual connectivity throughput. It was found that by utilizing $200 \mathrm{MHz}$ of bandwidth the application layer user throughput is surged to 206.7 Mbps.

\section{CONCLUSION}

This paper presents an overview of the dual connectivity feature for $5 \mathrm{G}$ user operating in non-standalone mode along with LTE. It is highlighted here that utilizing the existing street level dense micro sites is a viable solution for $28 \mathrm{GHz}$ outdoor propagation. The outdoor user typically stays in LOS with street micro sites and received signal does not experience high diffraction or blockage loss. The benefits and advantages of combining LTE with 5G NR, and using them simultaneously for improving the user experience through dual connectivity is demonstrated through the simulation results. It was found that user data rate and network capacity can be further enhanced by deploying the $5 \mathrm{G} \mathrm{NR}$ at $28 \mathrm{GHz}$ band with large available bandwidth. The higher frequency band operation was found effective in dense Manhattan grid environment particularly for outdoor users, mainly due to the LOS propagation.

\section{ACKNOWLEDGEMENT}

This research has been partially supported by the PriMO-5G project funded by the European Unions Horizon 2020 research and innovation programme under grant agreement No 815191, and also partially supported by Business Finland (under grants agreement No. 1916/31/2017 and 6388/31/2018).

\section{REFERENCES}

[1] M. Polese, M. Giordani, M. Mezzavilla, S. Rangan, and M. Zorzi, "Improved handover through dual connectivity in $5 \mathrm{~g}$ mmwave mobile networks," IEEE Journal on Selected Areas in Communications, vol. 35, no. 9, pp. 2069-2084, Sep. 2017.

[2] T. S. Rappaport, S. Sun, R. Mayzus, H. Zhao, Y. Azar, K. Wang, G. N. Wong, J. K. Schulz, M. Samimi, and F. Gutierrez, "Millimeter wave mobile communications for $5 \mathrm{~g}$ cellular: It will work!" IEEE Access, vol. 1, pp. 335-349, 2013.

[3] M. U. Sheikh, J. Säe, and J. Lempiäinen, "In preparation towards future cellular networks: the detailed analysis of macro and micro site densification and sector densification," Telecommunication Systems, vol. 65, no. 4, pp. 621-636, Aug 2017. [Online]. Available: https://doi.org/10.1007/s11235-016-0255-2

[4] R. Antonioli, G. Parente, C. Silva, D. Sousa, E. Rodrigues, T. Maciel, and F. Cavalcanti, "Dual connectivity for lte-nr cellular networks: Challenges and open issues," Journal of Communication and Information Systems, vol. 33, no. 1, Aug. 2018. [Online]. Available: https://jcis.sbrt.org.br/jcis/article/view/575

[5] H. Martikainen, I. Viering, A. Lobinger, and B. Wegmann, "Mobility and reliability in 1te-5g dual connectivity scenarios," in 2017 IEEE 86th Vehicular Technology Conference (VTC-Fall), Sep. 2017, pp. 1-7.

[6] 3GPP, "Release 15 description," 3rd Generation Partnership Project (3GPP), Technical Specification Group Services and System Aspects (Release 15), 032019.

[7] S. F. Yunas, M. Valkama, and J. Niemelä, "Spectral and energy efficiency of ultra-dense networks under different deployment strategies," IEEE Communications Magazine, vol. 53, no. 1, pp. 90-100, January 2015.

[8] E. Semaan, F. Harrysson, A. Furuskär, and H. Asplund, "Outdoor-toindoor coverage in high frequency bands," in 2014 IEEE Globecom Workshops (GC Wkshps), Dec 2014, pp. 393-398.

[9] 3GPP, "Study on channel model for frequencies from 0.5 to $100 \mathrm{ghz}$," 3rd Generation Partnership Project (3GPP), Technical Report (TR) 38.901, 12 2017, version 14.3.0. 